\title{
Carnets
}

Revue électronique d'études françaises de l'APEF

Deuxième série - 19 | 2020

Petite fabrique d'interprètes

\section{Nicolas Bouvier, Prométhée voyageur}

\section{Hugo Manuel Vaz}

\section{(2) OpenEdition}

Journals

Édition électronique

URL : http://journals.openedition.org/carnets/12072

DOI : 10.4000/carnets. 12072

ISSN : 1646-7698

Éditeur

APEF

\section{Référence électronique}

Hugo Manuel Vaz, « Nicolas Bouvier, Prométhée voyageur 》, Carnets [En ligne], Deuxième série - 19 2020, mis en ligne le 31 mai 2020, consulté le 22 décembre 2020. URL : http://

journals.openedition.org/carnets/12072 ; DOI : https://doi.org/10.4000/carnets.12072

Ce document a été généré automatiquement le 22 décembre 2020.

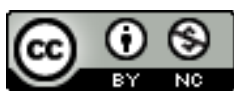

Carnets est mis à disposition selon les termes de la licence Creative Commons - Atribution - Pas d'utilisation commerciale 4.0 International. 


\title{
Nicolas Bouvier, Prométhée voyageur
}

\author{
Hugo Manuel Vaz
}

Ils avaient appris la vie dans les livres, et lui il

l'avait vécue.

Jack London, Martin Éden

1 Quand la tolérance assagit le fanatisme religieux, quand l'équité amenuise le rapport entre oppresseurs et opprimés, riches et pauvres, le seul clivage qui véritablement perdure entre les Hommes est celui qui existe entre nomades et sédentaires.

2 Selon Pierre Bonté, le sédentaire : «(...) résidant en un lieu qui reste central toute sa vie, même s'il bouge d'un lieu fixe à un autre. ${ }^{1}{ }^{1}$, s'oppose au nomade qui n'a pas d'habitation fixe et vit en déplacements continuels. On trouve cette opposition aussi bien dans les pages sacrées de la Bible que dans les livres d'Histoire.

Dans l'Ancien Testament, le livre de la Genèse raconte la tragique histoire de Caïn l'agriculteur sédentaire qui tue son frère Abel, le berger qui se déplace de pâturage en pâturage avec son troupeau. Le fratricide est motivé par la jalousie de Caïn, après que Dieu a refusé son offrande constituée de produits du sol et accepté l'offrande d'un premier-né du troupeau d'Abel. Dieu punit Caïn en le condamnant à l'errance suprême châtiment pour le sédentaire - :

Le Seigneur reprit : «Qu'as-tu fait ? La voix du sang de ton frère crie de la terre vers moi! Maintenant donc, sois maudit et chassé loin de cette terre qui a ouvert la bouche pour boire le sang de ton frère, versé par ta main. Tu auras beau cultiver la terre, elle ne produira plus rien pour toi. Tu seras un errant, un vagabond sur la terre. (Gen 4, 10-14).

4 C'est grâce à la sédentarité et à l'urbanité que les grandes civilisations se sont développées, sans parvenir, comme le montre l'Histoire, à éviter les conflits avec les nomades. Comme l'a bien noté Claude Riveline: "Les nomades nous ont toujours inspiré de l'horreur. Que l'on songe à Attila et ses Huns, épouvantables cavaliers derrière lesquels l'herbe ne repoussait pas, ou encore aux Sarrasins que Charles Martel arrêta à Poitiers en 732. » (Riveline, 1999).

Néanmoins, après les luttes et les invasions, les peuples nomades, les barbares, une fois 
vainqueurs, ont souvent fini par se sédentariser, au sein même parfois des villes et des contrées conquises.

De nos jours, l'appréhension envers le nomade, l'étranger, envers l'autre qui se déplace, existe encore, comme en témoignent les réactions xénophobes - contraires aux valeurs fraternelles des Droits de l'Homme - dans beaucoup de pays, par rapport aux tziganes, aux migrants économiques ou climatiques et aux réfugiés.

Dans nos sociétés modernes fortement urbanisées, on assiste à une progressive réduction des moments de sédentarité. Une élite urbaine se déplace continuellement aux quatre coins du monde en travail. D'un autre côté, le tourisme de masse a mis le monde à la disposition de millions de gens, qui visitent des pays qu'il faut voir. On vit dans l'extase de la mobilité. De nos jours, comme Claude Riveline l'a bien constaté :

Sédentaires et nomades cohabitent. Mais, plus encore, comme l'ont montré le philosophe Alain et Jean-Marc Oury, sédentarité et nomadisme cohabitent en chaque individu, chaque agent économique. En fait, le sédentaire a besoin de mouvement pour s'adapter et le nomade, à la différence de l'errant, sait préserver une permanence. (Riveline, 1999).

6 Mais l'errance, elle, n'est pas à la portée de tous. En général, on se borne à se rendre quelque part pour un laps de temps variable et on rentre chez soi. Le plus souvent, d'ailleurs, comme l'a dit Arthur Rimbaud : « On ne part pas » (Rimbaud, 1999 :180).

Rares sont ceux qui, un jour, comme Rimbaud, Gauguin, Brel ou l'écrivain voyageur Nicolas Bouvier osent tout quitter pour errer sur les routes inconnues du monde, vagabondant au gré des rencontres, des difficultés, des imprévus, du hasard.

En Occident, l'abandon au hasard est plutôt considéré comme une attitude négative car reflet de passivité. Toutefois, en Asie Centrale, cette attitude est respectée, car, ainsi, on est en accord avec le courant vital, avec le cours des choses tracé par Dieu. Le voyage y est connoté positivement, on lui associe même une mystique particulière, liée au pèlerinage religieux ou au commerce.

7 En juin 1953, à l'âge de 23 ans, Nicolas Bouvier quitte Genève au volant d'une fragile Fiat Topolino pour rejoindre, à Belgrade ${ }^{2}$, son ami peintre Thierry Vernet. Ensemble, ils vont entreprendre un long voyage (près de $7000 \mathrm{~km}$ ) à travers l'ex-Yougoslavie, la Turquie, l'Iran et l'Afghanistan.

Nicolas Bouvier avait déjà effectué de petits voyages en Italie, en Finlande et en Algérie. Mais, cette fois, il part pour un long périple, sans date de retour prévue, sans but véritable : « Le programme était vague, mais dans de pareilles affaires, l'essentiel est de partir.» (Bouvier, 1999: 10). L'élan même du voyage semble, au début, difficilement identifiable : «Un voyage se passe de motifs. Il ne tarde pas à prouver qu'il se suffit à lui-même ». (Bouvier, 1999 : 10).

8 L'Usage du Monde, publié en 1963, raconte la période du voyage de Nicolas Bouvier qui va de juin 1953 à décembre 1954, soit un an et demi de voyage. Le récit s'arrête, en Afghanistan, au Khyber Pass, à la frontière avec le Pakistan, après la séparation avec Thierry Vernet, effectuée à Kaboul. En effet, le peintre a décidé de partir pour l'île de Ceylan - actuel Sri-Lanka -, rejoindre sa fiancée Floristella Stéphani, et s'y marier.

Désormais, Nicolas Bouvier voyagera seul. Dans l'impossibilité de continuer vers l'Est, comme il le dit dans Le Poisson-scorpion : «Où irons-nous demain? Je m'étais attaché à cette école sans mensonges et, sans les interdits politiques, j'aurai continué vers l'Est par la Birmanie et le Sud chinois. » (Bouvier, 1991 : 19), il décide d'effectuer la descente du subcontinent indien, toujours en Fiat Topolino, jusqu'à Ceylan, où il arrive en mars 
1955, retrouvant Thierry Vernet et sa compagne.

Le dramatique séjour cingalais sera évoqué dans un récit envoûtant, Le Poisson-scorpion, écrit vingt-cinq années plus tard, et publié en 1981.

Après avoir vaincu les sortilèges de Ceylan et ses démons intérieurs, il poursuivra son voyage vers l'Est en embarquant, en octobre 1955, vers le Japon. Il y restera un an. Ce pays exercera sur lui une véritable fascination. Il évoquera l'univers japonais dans Japon (1967) et Chronique Japonaise (1975).

Nicolas Bouvier ne reviendra dans sa Suisse natale qu'après quatre années d'errance, de découvertes, d'épreuves et d'émerveillements.

Sa soif de voyager remonte à l'enfance : «C'est la contemplation silencieuse des atlas, à plat-ventre sur le tapis, entre dix et treize ans, qui donne ainsi l'envie de tout planter là. » (Bouvier, 1999: 10). Il a d'abord voyagé dans les pages des livres - les atlas mais aussi les romans d'aventure de Jack London, Jules Verne, Dumas, Stevenson - avant de voyager un jour dans les pages du livre du monde.

Sa fascination pour les images et les mots ne s'éteindra jamais. À Tabriz, en Iran, où les deux voyageurs restent six mois piégés par les neiges et le froid, Nicolas Bouvier contemple, émerveillé, une carte :

Avant de m'endormir, j'examinai la vieille carte allemande dont le postier m'avait fait cadeau : les ramifications brunes du Caucase, la tache froide de la Caspienne, et le vert olive de l'Orda des Khirghizes plus vaste à elle seule que tout ce que nous avions parcouru. Ces étendues me donnaient des picotements. C'est tellement agréable aussi, ces grandes images dépliantes de la nature... (Bouvier, 1999 : 157).

11 Thierry Vernet, qui connait bien son ami, lui enverra de Ceylan, une lettre pour l'appâter, en ces termes : « ...Ne serait-ce que pour te tenter, voilà les noms des bastions du fort: de l'Étoile, de la Lune, du Soleil, de Zwart, de l'Aurore, Pointe d'Utrecht, du Triton, de Neptune, de Clippenberg, et d'Éole. » (Bouvier, 1999 : 372).

Quand on part, le plus difficile c'est parfois de faire le premier pas, car on sait très bien ce que l'on quitte, mais on ignore ce que l'on va trouver : "Quand on part en voyage on se met en danger. " (Bouvier, 1999: 49). Lorsque Nicolas Bouvier rejoint à Belgrade Thierry Vernet, ce dernier est fatigué et déprimé. Il hésite à partir. Il raconte qu'en Slovénie, un aubergiste, comme pour le mettre en garde sur les difficultés du voyage, lui a dit : "Ich bin nicht verruckt, Meister, ICH bleibe zu Hause. ${ }^{3}$ (Bouvier, 1999 : 11). Bien sûr, Thierry Vernet se ressaisira, mais, il est vrai que pour oser faire un tel voyage, il faut avoir soit du courage, soit un petit grain de folie ! Ou un peu des deux, peut-être. Bien plus tard, à Tabriz, leur logeuse exprimera aussi une vision négative du voyage :

À mesure que la ville s'enfonçait dans l'épaisseur de l'hiver nous nous y trouvions mieux. Cette idée semblait tracasser la veuve Chuchanik, notre logeuse, qui nous rendait souvent visite : qu'on vienne - d'aussi loin et de plein gré - s'installer ici lui paraissait saugrenu. Au début, elle avait pensé que si nous étions ainsi sur les routes, c'était sûrement qu'on nous avait chassés de chez nous. (Bouvier, 1999 : 140)

12 La logeuse n'a pas totalement tort. Pour le sédentaire, le voyage s'assimile à l'exil, car le voyageur est séparé du pays natal et des êtres chers. Aux yeux des autres, où qu'il aille, où qu'il soit, il est étranger. À Prilep, en Macédoine, Nicolas Bouvier, avec humour, prend conscience de cette réalité : «Au point du jour, nous nous sommes retrouvés à la sortie de la ville avec quantité d'inconnus qui nous connaissaient - c'est ça être étranger. » (Bouvier, 1999 : 61).

Issu d'une famille bourgeoise très cultivée, Nicolas Bouvier semblait destiné à un avenir stable, une vie immobile, réglée comme une horloge suisse. En quittant Genève, il fuit 
les valeurs conventionnelles de son milieu social; il renonce à une carrière universitaire ; il tourne le dos à la morale protestante héritée de sa mère.

Pour Nicolas Bouvier, ce long périple dans l'espace et le temps représente une découverte du monde, une découverte d'autrui et une auto-découverte. Voyager vers l'Est, en direction au soleil levant, c'est voyager vers les pays qui ont vu l'éveil des civilisations et des croyances, vers cet Orient fondateur qui a toujours représenté, et représente encore, le mystère, le merveilleux, le raffinement, une certaine ascèse. Voyager vers l'Orient bien plus que voyager vers des pays, c'est voyager vers une idée, un concept, un paradigme, comme l'a bien exprimé Hermann Hesse :

... car notre but n'était pas simplement l'Orient, ou plutôt, notre Orient n'était pas seulement un pays et quelque chose de géographique, c'était la patrie et la jeunesse de l'âme, il était partout et nulle part, c'était la synthèse de tous les temps. (Hesse, 2005 : 39)

L'écrivain voyageur est aussi animé par le désir de transmettre ses découvertes. Sa quête existentielle est individuelle et altruiste. À plusieurs reprises dans L'Usage $d u$ monde - alors que Thierry Vernet peint ou dessine - Nicolas Bouvier, lui, écrit. À Tabriz, sa logeuse l'interpelle :

_ Mais que faites-vous donc ici?

_ J'ai ces élèves.

_ Mais le matin?

_ Vous voyez bien, je prends des notes, j'écris.

_ Moi aussi j'écris... l'arménien, le persan, l'anglais - fit-elle en comptant sur ses

doigts - ce n'est pas un métier. (Bouvier, 1999 : 140)

Pour la logeuse, l'écriture ce n'est pas très sérieux, il y a sûrement d'autres occupations bien plus utiles dans la vie ! À son retour en Suisse, c'est pourtant bien à l'artisanat de l'écriture que Nicolas Bouvier va se consacrer, travaillant, simultanément, en tant qu'iconographe.

Il va mettre plus de cinq ans à rédiger L'Usage du monde. De ce minutieux travail de maturation et de recherches résulte un texte dense, foisonnant de détails, de faits historiques et d'anecdotes.

Dans L'Usage du monde, après la préface, il y a un petit dossier, avec des lettres de Nicolas Bouvier, des photographies prises pendant le voyage, des projets de jaquettes de Thierry Vernet pour d'autres éditions du livre. Tous ces détails ancrent le récit dans le réel et sont aussi, selon Maria Hermínia Laurel : « comme une invitation - véritable incitation - à une lecture qui nous absorbe. » (Laurel, $2005: 2$ ).

Récit essentiellement chronologique, L'Usage du monde suit le flux du voyage en Asie réalisé par Nicolas Bouvier, voyageur/narrateur en mots, et Thierry Vernet, voyageur/ narrateur en dessins. Ses dessins un peu naïfs, aux noirs contours épais représentent des scènes du quotidien, des paysages, des animaux et reflètent la force et l'âpreté du monde qu'ils découvrent.

L'Usage du monde est un chant à deux voix qui résonnent en nous, s'unissant pour n'en faire qu'une, plus intense et plus ample.

Le récit relate et célèbre l'errance. Il révèle aussi un parcours individuel d'apprentissage - qu'on pourrait qualifier d'initiatique - qui se veut moralement utile. L'Usage du Monde peut être perçu comme un don fait à la communauté humaine - à tous ceux surtout qui ne sont pas partis, prisonniers de leurs mesquines habitudes proposant des réponses aux problèmes et aux maux de l'existence. 
Sous cette perspective, nous pouvons rapprocher - toutes proportions gardées -le geste de Nicolas Bouvier de celui de Prométhée, le titan bienfaiteur de l'Humanité, qui a dérobé le feu divin, le feu de la connaissance, pour le donner aux Hommes. Dans le dialogue Protagoras, Platon narre ainsi le larcin de Prométhée :

Il se glisse donc furtivement dans l'atelier commun où Athéna et Héphaïstos cultivaient leur amour des arts, il y dérobe au dieu son art de manier le feu et à la déesse l'art qui lui est propre, et il en fait présent à l'homme, et c'est ainsi que l'homme peut se procurer des ressources pour vivre. Dans la suite, Prométhée fut, dit-on, puni du larcin qu'il avait commis par la faute d'Épiméthée. ${ }^{4}$

Ulcéré, Zeus condamne Prométhée à un supplice atroce : il l'enchaine à un rocher et un aigle vient chaque jour dévorer son foie, celui-ci se régénérant durant la nuit.

Certes, Nicolas Bouvier ne va pas dérober sa connaissance du monde, une certaine sagesse, aux dieux, il les rapportera de son errance, enrichies par sa réflexion sur ce qu'il a vu, éprouvé et vécu. Toutefois, comme Prométhée, Nicolas Bouvier ne sortira pas indemne de son entreprise. Son voyage lui infligera une sanction qui, même si elle n'est pas aussi cruelle que la punition infligée par Zeus à Prométhée, n'en est pas moins réelle, indélébile.

En effet, le voyage n'est pas anodin : «On croit qu'on va faire un voyage, mais bientôt c'est le voyage qui vous fait, ou vous défait. » (Bouvier, 1999 : 10). Comme le suggère la dualité «fait/défait» - qui évoque un peu, en écho, l'incessant labeur du voyageur qui fait et défait ses bagages - le voyage est un processus dynamique de transformation et de réduction de l'être, comme Sarga Moussa l'a bien observé :

Être défait par le voyage, c'est à la fois être déconstruit et perdre une bataille. À toute une conception traditionnelle du voyage comme bonification du moi et accroissement des connaissances («les voyages forment la jeunesse»), Bouvier oppose un désapprentissage, une sorte de réduction volontaire du sujet voyageur ramené à ses justes proportions. ${ }^{5}$ circonstances et des expériences, un "moi" autre, plus vrai, libéré des anciennes valeurs bourgeoises qui l'écrasaient et faussaient sa vision de la vie. Au retour du voyage, le voyageur n'est plus tout à fait le même. Il y perd et il y gagne.

Le voyage a ses exigences, ses contraintes, ses dangers, ses douleurs, ses doutes et déroutes. Rien n'est donné. Les kilomètres doivent être gagnés grâce à des petits boulots: vente de dessins, conférences, leçons, articles. La Fiat Topolino doit constamment être réparée, démontée, remontée - c'est un travail de titan!

21 La frêle Fiat qui les relie à la vie, dans l'immensité désolée des pistes asiatiques, transforme le voyage à deux presqu'en un voyage à trois, tant elle est présente et exigeante. Elle est même personnalisée, parfois, comme à Ispahan, où Nicolas Bouvier se retrouve : «Assis sur le capot pour soulager la voiture malade... » (Bouvier, 1999: 231) ou lors de la terrifiante traversée du désert du Lout : « (...) on repartit vers Shurgaz avec cette voiture moribonde.» (Bouvier, $1999: 265)$. Les déboires subis par la voiture sont comme un reflet des difficultés que les voyageurs doivent eux aussi surmonter. Le voyage n'épargne ni les machines ni les hommes.

Étape après étape, les corps faiblissent, encaissent des coups, souffrent. Nicolas Bouvier raconte, par exemple, sa terrible blessure à la main, sur la route de Kaboul :

(...) la fièvre me rendait si maladroit que j'engageai la main gauche dans le ventilateur qui m'entailla quatre doigts jusqu'à l'os et m'envoya dinguer sur la route, le souffle coupé par la douleur. Thierry m'enveloppa la main dans des 
serviettes pour arrêter l'hémorragie, et c'est la seule occasion du voyage où la morphine que nous emportions trouva à s'employer. (Bouvier, 1999 :316) l'arrivée au Saki Bar où ils logent, d'un inquiétant voyageur, un «baladin » un peu sorcier, originaire du Sud de l'Inde. Le mystérieux visiteur professe une vérité amère (en italique dans le texte): «(...) l'homme est né pour errer, mourir, pourrir, être oublié. » (Bouvier, 1999 : 314). En quelques mots à peine, il vient de résumer la tragique 
destinée humaine. Sa seule présence suffit à mettre mal à l'aise les deux baroudeurs, car c'est toujours un peu angoissant d'être importunément confronté à l'évidence de la mort, à notre finitude.

Le voyage lui-même, qui est une succession d'arrivées et de départs s'apparente à la mort. Quand on part, on meurt un peu pour ceux qu'on quitte, on ignore si on les reverra un jour. C'est peut-être cela aussi voyager: apprendre à accepter la mort, apprendre à mourir.

Peu à peu, au fil des kilomètres parcourus, l'ancienne vie, l'ancien "moi" s'effacent et meurent eux aussi. Pour Nicolas Bouvier : "La vertu d'un voyage c'est de purger la vie avant de la garnir. » (Bouvier, 1999 : 25). Et de fait, le voyage le débarrasse des concepts vieillots qui encombraient sa tête. Il remet en question l'Occident, l'Europe, l'éducation reçue, la morale transmise par sa famille.

Il est à Belgrade, quand il s'en prend à l'enseignement prodigué, à l'étroitesse d'une " "culture" en pot ", domestiquée, éloignée du réel, un savoir enguirlandé :

Pendant mes années d'études, j'avais honnêtement fait de la «culture » en pot, du jardinage intellectuel (...) l'invention s'y confine en des fonctions décoratives et ne songe plus qu'à faire "plaisant», c'est-à-dire: n'importe quoi. Il en allait différemment ici ; être privé du nécessaire stimule, dans certaines limites, l'appétit de l'essentiel. (Bouvier, 1999 : 21).

À l'est d'Erzerum, en direction à la frontière entre la Turquie et l'Iran, il règle ses comptes avec son passé : famille, projets d'avenir, l'opinion des autres. Tout cela n'est pas réellement important, face à la vraie vie qu'est la découverte concrète du monde :

Finalement, ce qui constitue l'ossature de l'existence, ce n'est ni la famille, ni la carrière, ni ce que les autres diront ou penseront de vous, mais quelques instants de cette nature, soulevés par une lévitation plus sereine encore que celle de l'amour et que la vie nous distribue avec une parcimonie à la mesure de notre faible cœur. (Bouvier, 1999 : 110).

Le voyage poursuit son travail de déconstruction. En Iran, sur le site de Takht-e Djamshid, où Nicolas Bouvier collabore à des fouilles menées par une équipe française, il déglingue l'européocentrisme occidental qui fausse la perception du monde et de la vie :

(...) Alexandre, colon raisonnable apportant Aristote aux barbares; cette manie encore si répandue de vouloir que les Gréco-Romains aient inventé le monde; ce mépris - dans l'enseignement secondaire - des choses de l'Orient (...) (Bouvier, 1999 : 246)

Le voyage est à la fois mort et résurrection. L'écrivain voyageur a fait le ménage dans sa tête et désormais il est sûr de lui, de son choix de vie errante. Voyager et découvrir le monde, aller au contact des autres, le corps et l'esprit (al)légers, les sens vifs et l'esprit alerte, c'est la seule façon de vivre qui lui convienne. Vivre, c'est être en mouvement.

On a l'impression que Nicolas Bouvier et Thierry Vernet ne glissent pas sur le monde, mais qu'ils s'y écorchent et s'y accrochent, comme s'ils ne voulaient pas en perdre une seule miette. L'errance se fait en roulant très lentement. Le flâneur bienheureux déclare, en route vers la Macédoine : « Nous nous refusions tous les luxes sauf le plus précieux : la lenteur » (Bouvier, $1999: 49)$.

31 La luxueuse et royale lenteur du voyage permet de ménager la fragile voiture, d'éviter ou de surmonter les dangers de la route, mais surtout elle favorise la contemplation du monde, l'observation de détails que seul le temps qu'on prend permet de discerner. Quand on est trop pressé, on reste à la superficie lisse des choses. Pour arriver à mieux 
voir, à comprendre ce que l'on voit, il faut commencer par regarder le monde et les autres sans hâte, les yeux et l'esprit bien ouverts.

À maintes reprises Nicolas Bouvier évoque le bonheur provoqué par le spectacle du monde surtout au début du voyage, qui est très jouissif. À Belgrade, totalement absorbé par la vie, il constate : «Si je n'étais pas parvenu à y écrire grand-chose, c'est qu'être heureux me prenait tout mon temps.» (Bouvier, 1999: 44). En Turquie, à Sungulu, il note: «Ces grandes terres, ces odeurs remuantes, le sentiment d'avoir encore devant soi ses meilleures années multiplient le plaisir de vivre comme le fait l'amour.» (Bouvier, 1999: 90). Leur vie est devenue si plaisante et enivrante qu'elle acquiert une dimension aristocratique : à Belgrade, Nicolas Bouvier affirme : «D'ailleurs, nous étions rois.» (Bouvier, 1999 : 33) et, à Chiraz, il évoque même : «(...) la vie de seigneurs » (Bouvier, 1999 : 243).

Disponibles au monde et disponibles aux autres, les deux voyageurs cherchent l'émotion née du contact avec les gens.

Nicolas Bouvier démontre une grande passion pour la Serbie et ses habitants, il assure qu'« Il y a, en Serbie, des trésors de générosité personnelle.» (Bouvier, 1999 : 20). Dans ce pays qu'il aime tant, il a vécu des moments extraordinaires, comme cette nuit magique passée dans un camp tzigane - frères errants à l'esprit libre, musiciens dans l'âme, dans lesquels, bien-sûr, Nicolas Bouvier se revoie - à Bogoiévo, au nord de Belgrade, où les deux voyageurs sont allés enregistrer des chansonssur le magnétophone emporté dans leurs bagages. Les poignants chants tziganes l'émeuvent :

De vieilles complaintes que leurs cousins des villes ont oublié depuis longtemps. Des chansons frustes, excitées, vociférantes qui racontent en langue romani les avatars de la vie quotidienne: larcins, petites aubaines, lune d'hiver et ventre creux... (Bouvier, 1999: 37)

Le voyage est aussi une découverte musicale. Dans L'Usage du monde, Nicolas Bouvier s'intéresse beaucoup au folklore, à la musique et aux chants des peuples qu'il découvre, surtout en Serbie, en Macédoine, et en Iran. À Téhéran, il note : «À la radio résonne cette belle musique persane de tar, ancienne, semblable à celle d'un Ségovia détaché de tout, semblable aussi à un peu de verre brisé qui dégringole avec indolence. » (Bouvier, 1999 : 232). Même admiration, à Quetta, quand l'étonnant baladin indien interprète une chanson qui, comme la madeleine de Proust, éveille le souvenir des chants entendus dans les pays balkaniques dans une riche synesthésie auditive, olfactive et visuelle :

Sortes de soupirs chantés qui rappelaient de façon saisissante les chansons sveda de Bosnie. Nous retrouvions l'odeur des piments rouges, les tables sous les platanes de Mostar ou de Sarajevo, et les tziganes de l'orchestre dans leurs complets limés (...). (Bouvier, 1999 :314).

La musique ne connait pas de frontière ; voyageuse, elle aussi erre au gré des routes, des voyages, des rencontres et des migrations. La fascination de Nicolas Bouvier pour la musicalité des mots semble se prolonger dans sa passion pour la musique. Enfant, il a d'ailleurs suivi des leçons de musique et il sait jouer de quelques instruments. Thierry Vernet lui-même est musicien et a emporté dans ses bagages un accordéon. Durant le voyage, ils joueront parfois de la musique, soit lors de moments de fête, soit dans des diners où ils sont invités, soit même en tant qu'attraction musicale internationale au Saki Bar à Quetta, en Iran, pour gagner un peu d'argent.

La musique est importante car, étant un langage universel, l'émotion qu'elle véhicule abat les murs de la diversité de langues et de cultures et favorise la convivialité. En Macédoine, Nicolas Bouvier constate, amusé, le pouvoir magique de la musique : « Ici, 
comme en Serbie, la musique est une passion. C'est aussi un "Sésame" pour l'étranger: s'il l'aime, il aura des amis. S'il enregistre tout le monde, même la police, s'emploiera à lui racoler des musiciens. » (Bouvier, 1999 : 72).

Comme la musique, le rire et l'humour permettent de vaincre les barrières linguistiques et d'établir un lien avec les gens. Les voyageurs feront parfois usage de ce que l'on peut appeler la fraternité du rire pour se tirer d'affaire. Nicolas Bouvier se rend compte du pouvoir du rire quand, à Téhéran, face au directeur de l'Institut francoiranien peu enclin à accepter leurs services de voyageurs artistes, Thierry Vernet est soudain pris d'un fou rire contagieux, qui détend l'atmosphère et renverse la situation en leur faveur :

[Le directeur] se mit donc à rire plus fort que nous, habilement d'abord, par gammes bien dosées, puis pour de bon. Quand la secrétaire entrebâilla la porte. Il

lui fit signe d'apporter trois verres, et quand nous eûmes repris souffle tout était devenu différent. (Bouvier, 1999 : 215-216).

Cet épisode lui servira de leçon. Il confesse : «Je n'oubliais pas que c'est sur un éclat de rire que le vent avait tourné pour nous. Depuis j'ai toujours en réserve quelque chose de cocasse à me murmurer intérieurement quand les affaires tournent mal... » (Bouvier, 1999 : 217).

Le vagabondage en Asie Centrale n'est pas une avancée totalement libre, continue, effrénée. Le voyage est entrecoupé de moments de sédentarité qui peuvent durer quelques jours, quelques semaines ou six mois, comme à Tabriz, à cause par exemple, des rigueurs du climat; des maladies qui les frappent; des nombreuses réparations que la voiture exige; des petits boulots exercés afin de gagner un peu d'argent qui alimentera les ventre creux et l'errance; du repos qu'il faut s'octroyer après des étapes éprouvantes. Ces pauses sont des moments privilégiés pour partager l'existence, souvent austère et frugale - parfois même misérable - des gens ; apprécier leur savoirvivre; prendre le pouls de la situation économique et sociale du pays où ils se trouvent; flâner à pied dans les villes et les villages, visiter des monuments, des mosquées, des musées pour essayer de mieux cerner les cultures locales ; et bien-sûr, prendre le temps de peindre, lire et écrire.

Nicolas Bouvier s'intéresse profondément aux gens, il cherche réellement à les connaitre et à les comprendre. Il n'hésite pas à se rendre à leur rencontre. Il les observe sans juger, sans récrimination, avec bienveillance, même si parfois elle est teintée d'une fine ironie, mais jamais nous n'avons décelé de mépris, de méchanceté, de supériorité ou d'arrogance. L'autre est d'abord un frère humain, quelle que soit son origine, sa religion ou son statut social. S'il se montre critique et parfois corrosif c'est surtout face à l'Occident, à l'Europe et aux quelques occidentaux auxquels il a affaire.

Grâce à ce vivre ensemble, cette proximité, ce partage, Nicolas Bouvier va vivre des situations émouvantes. Par exemple, à Tabriz, dans une gargote minable où il se rend fréquemment pour passer le temps ou essayer d'écrire, à midi, des portefaix encore plus miséreux que lui, viennent prendre leur maigre repas constitué d'un bout de pain, de sucre et de thé, que, cependant, ils n'hésitent pas à partager. L'écrivain voyageur registre ce geste de touchante humilité et générosité :

Jamais quand j'étais à leur table, ils ne commençaient sans m'offrir d'abord: Beffarmâid - c'est à vous - cette minable pitance qui s'en trouvait sanctifiée. Si j'acceptais, c'en était fait du repas de la journée. Je me demandais quel ordre poussait ces ventres-creux à offrir aussi machinalement le peu qu'ils possèdent ? Un ordre noble en tout cas ... (Bouvier, 1999 : 156). 
Toujours à Tabriz, Nicolas Bouvier s'aperçoit que les timbres des lettres qu'il a reçues d'Europe ont été dérobés. Il se plaint à une vieille mégère. Grand émoi ; le quartier s'agite; des claques sifflent; des garnements sanglotent et, bientôt, comme par enchantement, les timbres lui sont rendus. Ce larcin prestement résolu motive cette réflexion morale :

La loi du quartier (...) avait été enfreinte, et cette loi commande d'être honnête, dans les petites choses surtout, qui relèvent du quotidien et de la conduite. On a plus de latitude dans les grandes qui appartiennent au destin. (Bouvier, 1999 : 191)

En Turquie, après un pénible séjour effectué à Istanbul, en route vers Erzerum, dans l'arrière-pays, il reconnait, la noblesse des valeurs qui régissent l'existence des villageois, souvent très démunis, qu'il croise: «L'hospitalité, l'honnêteté, le bon vouloir, un chauvinisme candide sur lequel on peut toujours faire fond : voilà les vertus qu'on trouve ici. Elles sont simples et bien palpables.» (Bouvier, 1999 : 101).

En quittant l'Iran, il souligne la qualité de l'accueil qui leur a été fait à Téhéran : « Ici, où tout va de travers, nous avons trouvé plus d'hospitalité, de bienveillance, de délicatesse et de concours que deux persans en voyage n'en pourraient attendre de ma ville où pourtant tout va bien. (Bouvier, 1999 : 229).

41 Après la traversée du désert du Lout, le séjour en région baloutche, à Quetta - qui aujourd'hui intègre le Pakistan - permet au voyageur d'apprécier la ville et l'austère et droit savoir-vivre des habitants. Il note en arrivant: "Il y a peu de misère ici, et beaucoup de cette frugalité qui rend la vie plus fine et plus légère que cendre. (Bouvier, 1999 : 277). Après avoir passé de longues heures au Ramzan Garage où la Fiat Topolino est réparée par d'habiles mécaniciens, il affirme : «Impossible de travailler avec eux sans s'en faire des amis. (Bouvier, 1999: 282). Il trace un portrait flatteur des Baloutchs : Sous les misérables haillons, il perçoit le scintillement d'une âme admirable. Comme souvent, il faut prendre le temps de scruter au-delà de l'apparence pour découvrir une vérité cachée :

Le Baloutch est plutôt sûr de lui. Son aisance morale éclate dans ce sourire qui flotte à hauteur de barbe et dans le drapé de hardes toujours propres. Il est très hospitalier et rarement importun. (...) Les Baloutchs sont musulmans sunnites, sans trace de fanatisme. (Bouvier, 1999 : 285)

En voyageant à travers l'Asie Central au contact du monde et des autres, dans des pays où les gens vivent d'une manière si austère, Nicolas Bouvier prend conscience du cadre paradoxal de sa quête existentielle.

À Bayburt, un misérable village turc perdu dans l'Anatolie, où tout manque, il constate la fascination des villageois pour les produits technologiques occidentaux. Alors que les deux voyageurs attendent de l'Orient une aide spirituelle pour répondre à des problèmes existentiels, les pauvres villageois turcs attendent de l'Occident une aide matérielle pour améliorer leurs effroyables conditions de vie. Nicolas Bouvier résume ainsi la situation: "Nous comptons sur leurs recettes pour revivre, eux sur les nôtres pour vivre. (Bouvier, 1999: 103). Chacun a ses manques qu'il espère un jour pouvoir combler.

À aucun moment, dans L'Usage du monde, Nicolas Bouvier ne manifeste de regrets pour être parti, malgré les rudes épreuves endurées, certains moments de découragement, et même de désarroi, comme celui vécu à Ispahan, en Iran. Ce jour-là, les deux amis se retrouvent dans un lieu inquiétant, qui est comme ensorcelé. Les voyageurs, déconcertés, font promptement demi-tour. Selon Nicolas Bouvier, il y a: «(..) des 
paysages qui vous en veulent et qu'il faut quitter immédiatement sous peine de conséquences incalculables il n'en existe pas beaucoup, mais il en existe. Il y en a sur cette terre cinq ou six pour chacun de nous. » (Bouvier, 1999 : 235).

Il faudra attendre le séjour traumatique sur l'île de Ceylan, pour qu'il manifeste la nostalgie de la distante Europe. Il confesse, en lisant une lettre venue d'Europe : «J'y ai trouvé cette phrase : «Au nord de Grenoble il pleuvait. L'herbe était haute. Il y avait des geais dans les noyers ", qui m'a étrangement remué. À cause de l'herbe. (...) L'Europe peut avoir bien des défauts, mais elle a une herbe incomparable... ». (Bouvier, 1991 : 147).

Quant à Thierry Vernet, sans doute psychologiquement plus fragile, il craque durant l'interminable enfer blanc de Tabriz. Il dit à son ami: «Je n'en peux plus de cette prison, de cette trappe (...) regarde où nous en sommes après huit mois! piégés ici. » (Bouvier, 1999: 156). C'est à ce moment-là qu'il décide d'arrêter le voyage, d'abandonner Nicolas Bouvier et de partir pour Ceylan pour retrouver sa fiancée.

La séparation des deux amis voyageurs a lieu à Kaboul. Aux difficultés du voyage s'ajoute alors, pour Nicolas Bouvier, celle de voyager seul. C'est difficile de perdre un compagnon d'errance, même s'il semble accepter sa décision : «Bon. Je ne voyais que la maladie ou l'amour pour interrompre une telle entreprise. Je préférais que ce fût l'amour. Il poussait sa vie. J'avais envie d'égarer la mienne... » (Bouvier, 1999 : 157).

Mais, après la séparation, le ton du récit change légèrement. L'enthousiasme vital de la jouissive et épicurienne flânerie à travers l'éden des Balkans semble maintenant très loin. Le majestueux massif de l'Hindou-Kouch, qui s'étend à perte de vue, est plus propice à la méditation.

Mais l'essentiel, c'est d'aller de l'avant, de poursuivre le vagabondage, au volant de la toujours vaillante Fiat Topolino. Pour Nicolas Bouvier, bouger, c'est une nécessité vitale. Il l'a dit: "J'ai trop besoin de cet appoint concret qu'est le déplacement dans l'espace» (Bouvier, 1999: 51). Il craint trop l'immobilité, les grilles des prisons de l'existence normative, des habitudes qui ankylosent et dont il faut se méfier : "C'est si doux les vieilles habitudes, même celles qui vous oppriment. » (Bouvier, 1999 : 107).

Cette immobilité tant redoutée, c'est aussi une des facettes du châtiment infligé par Zeus à Prométhée. En l'enchainant à un rocher, à la matière rugueuse et inerte, il prive Prométhée du mouvement et l'isole du monde. Prométhée est en fait triplement condamné : à l'immobilité, à la souffrance et à la solitude.

47 Après la montée éprouvante du Kybber-Pass, Nicolas Bouvier se retrouve seul au sommet du col. Il contemple le paysage, perdu dans ses pensées : «J'ai passé une bonne heure immobile, saoulé par ce paysage apollinien.» (Bouvier, 1999 : 373). Suite à cette longue et intense contemplation, il a une sorte d'illumination :

Ce jour-là, j'ai bien cru tenir quelque chose et que ma vie s'en trouvait changée. Mais rien dans cette nature n'est définitivement acquis. Comme une eau le monde vous traverse et pour un temps vous prête ses couleurs. Puis se retire, et vous replace devant ce vide qu'on porte en soi, devant cette insuffisance centrale de l'âme, qu'il faut bien apprendre à côtoyer, à combattre, et qui, paradoxalement est notre moteur le plus sûr. (Bouvier, 1999 : 374)

Face au paysage grandiose, Nicolas Bouvier est confronté au "vide qu'on porte en soi ", au vide de l'âme, au vide de l'univers, au sens insondable de l'existence, ou, comme l'a exprimé Fernando Pessoa, à : «L'immensité vide des choses, le vaste oubli qui règne dans le ciel et sur la terre... » (Pessoa, 1999 : 209). Et pourtant, c'est précisément ce silencieux gouffre intérieur, "cette insuffisance centrale de l'âme", qui motive le 
voyageur à poursuivre le voyage dans sa quête, à avancer, à la recherche du neuf, du changeant, de l'inattendu, de soi, pour, peut-être, arriver un jour à combler le gouffre de l'âme, le vide intérieur entrevu. L'âme du voyageur n'a pas encore trouvé le repos.

Heureusement, apportées par le vent: «(...) le voyageur reçoit par bouffées l'odeur mûre et brûlée du continent indien...» (Bouvier, 1999: 376). C'est le souffle d'un continent inconnu qui déjà l'appelle, lui fait signe, lui tend les bras.

Le récit ne se termine pas par un point final, mais par trois points de suspension qui signalent la poursuite du voyage et de la quête, car ils contiennent en eux tous les rêves du voyageur, à la veille d'un nouveau départ.

Amin Maalouf Malouf distingue deux sortes de voyageurs :

Bien des hommes découvrent le vaste monde en cherchant seulement à faire fortune. Quant à toi, mon fils, c'est en cherchant à connaître le monde que tu trébucheras sur un trésor. (Maalouf, $2016: 267$ )

51 Nicolas Bouvier fait indubitablement partie de ces voyageurs qui s'en vont «en cherchant à connaître le monde ", à connaître les autres, à se connaître.

Si Prométhée a volé aux dieux le feu divin pour le donner aux hommes, Nicolas Bouvier, quant à lui, a rapporté de son errance en Asie Centrale une sagesse austère, une vérité existentielle dont, lui aussi, nous fait don.

Invitation au voyage, invitation à une méditation sur l'existence, de L'Usage du monde, le lecteur peut faire usage, doit faire usage, en extrayant du récit sa transmissible quintessence.

\section{BIBLIOGRAPHIE}

\section{Bibliographie citée}

BONTÉ, Pierre (2019). Nomades et sédentaires : conflits multiples et symbioses dans l'Histoire. [Version en ligne consultée le 16 mars 2020] URL :http://www.leconflit.com/2019/09/nomades-et-

sedentaires-conflits-multiples-et-symbioses-dans-l-histoire.html

BOUVIER, Nicolas (1991). Le Poisson-scorpion. Paris : Payot.

BOUVIER, Nicolas (1999). L'Usage du monde. Genève : Librairie Droz.

HESSE, Hermann (2005). Le Voyage en Orient. Paris : Le Livre de Poche.

HESSE, Hermann (2017). Le Loup des Steppes. Paris : Le Livre de Poche.

LA BIBLE Version proposée sur le site de l'Association Épiscopale Liturgique pour les pays

Francophones : https://www.aelf.org/bible/Gn/4 [Version en ligne consultée le 18 mars 2020]

LAUREL, Maria Hermínia (2006). « De l'usage du monde comme itinéraire identitaire », Cadernos de Literatura Comparada, 14/15, pp. 185-207.

PESSOA, Fernando (1999). Le Livre de l'Intranquillité de Bernardo Soares. Paris : Christian Bourgeois Éditeur.

MAALOUF, Amin (2016). Léon l'Africain. Paris : Le Livre de Poche. 
MOUSSA, Sarga (2004). « Nicolas Bouvier ou la réinvention du voyage en Orient au XXe siècle » in Seuils et Traverses 4, actes du colloque d'Ankara des 2-4 juillet 2003, Emin Özcan (dir.), Ankara Üniversitesi Basimevi, pp. 164- 176. [Version en ligne consultée le 20 mars 2020] URL : https:// halshs.archives-ouvertes.fr/halshs-00257255/document

PLATON, Protagoras, https://beq.ebooksgratuits.com/Philosophie/Platon-Protagoras.pdf [Version en ligne consultée le 18 mars 2020]

RIMBAUD, Arthur (1999). Poésies. Une Saison en Enfer, Illuminations. Paris : Éditions Gallimard.

RIVELINE, Claude (1999). Nomades et sédentaires, l'irréductible affrontement, Le Journal de l'École de Paris, $\mathrm{n}^{\circ}$ 16, mars-avril, pp. 41- 47. [Version en ligne consultée le 16 mars 2020] URL http:// riveline.net/nomades.pdf

\section{Bibliographie de référence}

JACQUIER, claire (1980). « Le voyage de l'allégement ou les chances de l'inconfort intellectuel. Un récit de Nicolas Bouvier: L’usage du Monde, Études de lettres, Lausanne, nº 4, pp. 57-67.

MAGAZINE LITTÉRAIRE, Les Écrivains voyageurs - de l'aventure à la quête de soi, no 432, Juin 2004.

MAGETTI, Daniel (1989). « Mots d'ordre et ordre de mots, Poésie et prose dans l'œuvre de Nicolas Bouvier », Etudes de Lettres, juillet-septembre, nํ3, pp. 79-88.

MAGETTI, Daniel (1991). « Nicolas Bouvier, voyageur et moraliste », Versants Littératures Romanes en Suisse, $\mathrm{n}^{\circ} 20$, pp. 83-92.

LONDON, Jack (2017). Martin Éden. Paris : Librairie Hachette.

\section{Site web consulté}

https://www.rts.ch/archives/dossiers/3477393-nicolas-bouvier-ecrivain-et-voyageur.html (On y trouve de très intéressantes archives audiovisuelles sur Nicolas Bouvier)

\section{NOTES}

1. Bonté, Pierre, Nomades et sédentaires: conflits multiples et symbioses dans l'Histoire. http:// www.leconflit.com/2019/09/nomades-et-sedentaires-conflits-multiples-et-symbioses-dans-1histoire.html [Version en ligne consultée le 16 mars 2020]

2. Belgrade était alors la capitale de la Yougoslavie. Après les fratricides et tragiques conflits qui ont conduit à la dissolution du pays pendant la dernière décennie du $\mathrm{XX}^{\text {ème }}$ Siècle, Belgrade est devenue, à partir de 2006, la capitale de la Serbie.

3. En allemand dans le texte. « Je ne suis pas fou, Maître, je reste à la maison ».

4. Platon, Protagoras https://beq.ebooksgratuits.com/Philosophie/Platon-Protagoras.pdf (p 50) [Version en ligne consultée le 18 mars 2020]

5. Moussa, Sarga, Nicolas Bouvier ou la réinvention du voyage en Orient au XXe siècle, https:// halshs.archives-ouvertes.fr/halshs-00257255/document (p 6) [Version en ligne consultée le 20 mars 2020] 


\section{RÉSUMÉS}

L'opposition entre nomades et sédentaires remonte à la nuit des temps. En Occident, le nomade est souvent redouté, mais, en Asie, il a une aura noble, comme le découvre Nicolas Bouvier, écrivain voyageur d'origine suisse, qui, dans les années cinquante, part avec Thierry Vernet à la découverte de l'Asie Centrale, des autres, de lui-même et des valeurs essentielles. Leur errance asiatique, périple jouissif mais rempli d'embuches et de souffrances, se caractérise par la lenteur, l'apprentissage du dénuement, l'importance des rencontres, du partage, la méditation sur les valeurs de la vie. Le voyage durera presque deux ans et sera la matière du livre L'Usage du monde. Tel Prométhée qui a dérobé le feu divin, le feu de la connaissance, pour le donner à la communauté humaine, Nicolas Bouvier, en faisant le compte-rendu de sa lente et heureuse flânerie, nous fait cadeau des découvertes glanées au gré des routes, d'un savoir caché, d'une sagesse austère.

The opposition between nomads and sedentary people goes back to the dawn of time. In the West, the nomad is often feared, but in Asia, he has a noble aura, as discovered by Nicolas Bouvier, a writer-traveller of Swiss origin, who, in the 1950s, set off with Thierry Vernet to discover the Central Asia, others, himself and essential values. Their Asian wandering, a joyful journey but full of pitfalls and suffering, is characterized by slowness, learning about deprivation, the importance of encounters, sharing, meditation on the values of life. The journey will last almost two years and will be the subject of the book L'Usage du monde. Like Prometheus who stole the divine fire, the fire of knowledge, to give it to the human community, Nicolas Bouvier, by giving an account of his slow and happy wandering, gives us the gift of discoveries gleaned along the way, of hidden knowledge, of austere wisdom.

\section{INDEX}

Mots-clés : voyage, Bouvier (Nicolas), existence, Orient, Prométhée

Keywords : travel, Bouvier (Nicolas), existence, East, Prometheus

\section{AUTEUR}

HUGO MANUEL VAZ

moraisvaz[at]sapo.pt 